\title{
Primary Hyperparathyroidism - Case Report of a Female Patient with Hypercalcemic Crisis
}

\author{
Rizwan Khalid ${ }^{1 *}$, Sidra Rizwan ${ }^{1}$ \\ ${ }^{1}$ Assistant Professor Surgery, Central Pak Medical College Lahore, Pakistan.
}

*Corresponding Author: Rizwan Khalid, Assistant Professor Surgery, Central Pak Medical College Lahore, Pakistan.

\section{Received: 16 August 2021 | Accepted: 28 August 2021 | Published: 10 September 2021}

Citation: R Khalid, S Rizwan. (2021). Primary Hyperparathyroidism - Case Report of a Female Patient with Hypercalcemic Crisis. Endocrinology and Disorders. 5(6): DOI:10.31579/2640-1045/087

Copyright: () 2021 Rizwan Khalid, This is an open-access article distributed under the terms of the Creative Commons Attribution License, which permits unrestricted use, distribution, and reproduction in any medium, provided the original author and source are credited.

\begin{abstract}
:
Primary hyperparathyroidism is a result of increased and uncontrolled function of the parathyroid hormone caused by hyperfunction of one or more parathyroid glands. The cause of hyperfunction of the parathyroid glands could be adenoma, hyperplasia, and carcinoma. Primary Hyper parathyroidism is the most common cause of Hypercalcemia in outpatient population.

The paper presents the case report of a 60years old female treated in our Hospital in March 2013. She presented in outpatient department with complaints of joints pain, Hypertension and renal failure for last 8years. After clinical, laboratory and radiological examination she was diagnosed as a case of PHPT due to a Giant Parathyroid Adenoma.

After supportive, symptomatic and surgical treatment, the patient was back to normal daily activities without any signs of disease.

Key words: hypercalcemia; hyperparathyroidism; adenoma
\end{abstract}

\section{Introduction}

Primary hyperparathyroidism occurs as a result of increased and uncontrolled secretion of parathyroid hormone because of hyperfunction of one or more parathyroid glands. The cause of hyperfunction of parathyroid glands is, in majority of cases, adenoma, followed by hyperplasia, and carcinoma only in 1 to $2 \%$ of cases [1].

The frequency of primary hyperparathyroidism is $1 / 1000$ individuals in general population $[1,2]$. In patients with primary hyperparathyroidism of benign etiology, the ratio of female/male is $3: 2$ [3]. The main sign of disease is Hypercalcemia caused by an increased resorbtion of calcium from bones, decreased urinary elimination of calcium and an increased absorption of calcium in bowels.

In patients with primary hyperparathyroidism, calciuria has been reported, with an increased tendency to urolithiasis that can lead to Renal Failure and Hypertension, then polyuria because of an osmotic diuresis which leads to dehydration and loss of weight [1-3].

In the clinical presentation, the following complains were noted: poor appetite, nausea accompanied by vomiting, loss of weight, constipation, pain in bones, symptoms of nephrolithiasis, emotional instability, and depression. Clinical sings depend on the parathormone values, as well as the level of Hypercalcemia [1-3].
Diagnosis was established based on History, objective examination, laboratory analysis, raidioimmunological evaluation of PTH \& Calcium in serum, Radiological examination of Parathyroid glands.

Surgical therapy remains the only definite and durable treatment for parathyroid adenoma and there is a clear paradigm shift towards focused Parathyroid surgery (the exploration of a single site of suspected Parathyroid Adenoma) guided by Radiological Studies [4].

\section{Case Report}

A 60 years old Female, Admitted in East surgical Ward Mayo Hospital Lahore in March 2013 with Suspicion of Parathyroid Adenoma. Her detailed history showed that she had complaints of joints pain for the last 8 years and was treated for arthritis in the form of painkillers and Calcium supplements but of no avail. For the last 25 days she was almost confined to bed. She developed hypertension secondary to renal failure caused by nephrolithiasis. She also noticed epigastric pain, nausea, anorexia and weight loss.

She was investigated for nephrolithiasis and was found Hypercalcemia in a specialized hospital and upon suspicion of Primary Hyperparathyroidism she was referred to us.

On clinical examination we found high blood pressure and a mass on rt. Side of neck in the anterior triangle extending from clavicle to below the 
thyroid cartilage and between the trachea and sternocledomastoid muscle It moves on deglutition, firm in consistency, non-tender, mobile, free of overlying skin.

Laboratory Analysis showed CBC: HB- 10.6, WBC- 9600, HCT- 30, PLT- 169000 LFT: Billirubin total-0.5, SGPT-25, SGOT-19, ALP-126, TOTAL PROTEIN-7, ALBUMIN-3.9, HBsAG-NEG, ANTI HCV- NEG PTH $>2500 \mathrm{pg} / \mathrm{ml}$ (NormalValue $11 \mathrm{pg} / \mathrm{ml}-67 \mathrm{pg} / \mathrm{ml}$ ) $24 \mathrm{hrs}$ Urine Calcium $270 \mathrm{mg} / 24 \mathrm{hr}$ (Normal Value 100mg-300mg/24hr) 25 HYDROXYVITAMIN $\quad$ D- $15.02 \quad \mathrm{ng} / \mathrm{ml}$ Serum Calcium 20.83..14.58..13.37mg/dl (Normal Value $8.5 \mathrm{mg} / \mathrm{dl}$ $10.5 \mathrm{mg} / \mathrm{dl}$ )Urea $78 . .83 . .92 \mathrm{mg} / \mathrm{dl}$ (Normal Value $15-30 \mathrm{mg} / \mathrm{dl}$ )Creatinine 2.4..3.0..3.6..2.9mg/dl (Normal Value less than $1 \mathrm{mg} / \mathrm{dl}$ ) S/Potassium 5.1..4.4..5.0..4.1 mg/dl... (Normal Value $3.5-4.0 \mathrm{mg} / \mathrm{dl}$ ) Ultrasonography Neck showed Right inferior parathyroid adenoma $7.2 \mathrm{~cm} \times 1.8 \times 2.29 \mathrm{~cm}$ in dimension is seen reaching almost the medial clavicular end. A Spindle shaped $5 \mathrm{~cm}$ long and $1 \mathrm{~cm}$ thick prevertebral mass is seen pushing the esophagus anteriorly, its inferior edge could not be ascertained. This tubular structure is vascular on Doppler. Suspicion of A Leiomyoma?

MRI Neck Showed Nodule at the posterior aspect of the rt. Thyroid lobe(present in the rt. Parathyroid) and Elongated soft tissue mass, predominantly along the rt. Side of neck and extending to prevertebral space without involvement of surrounding soft tissues or erosion of vertebra- favors the MRI finding of benign mass, most likely Leiomyoma. Ultrasonography Abdomen Showed both kidney are normal in size and shape. Both shows increased cortical echogenicity with reduced corticomedullary differentiation- suggestion of renal parenchymal disease. A calculus measuring $5.5 \mathrm{~mm}$ seen at the upper pole of left kidney, no hydronephrosis seen in either side X-Rays of Long bones and skull showed decreased bone density.

Preoperatively she was treated with Injection Pamidronate 60mg ,Inj. Lasix 40mg X BD,Inj. Calcitonin 200ugm/IU X BD ,Inj. NS 1000ml X TDS \& Tab. Norvasc 5mg X OD for Symptomatic Relief Excision of right parathyroid adenoma \& right lobectomy and is thmusectomy was Done and Histopathology Report showed Parathyroid adenoma size $7 \mathrm{~cm}$. In the postoperative period, the patient felt much better. She had good appetite, she was gaining weight, and stools were regular. Post Operatively PTH $51.10 \mathrm{pg} / \mathrm{ml}$, BUN 23, CREATININE 2.38, ALKALINE PHOSPHATASE 1081, SERUM CALCIUM 8.87 .

\section{Discussion}

The effect of excessive amounts of parathormon is best seen on the cells of renal tubules, bones, and digestive tract mucous membrane. Its effect on tubular epithelium causes increased phosphaturia, kaliuria and natriuria, with retention of calcium, magnesium and hydrogen, which results in hypophosphathemy and Hypercalcemia [4]. Primary hyperparathyroidism is a rare disease which should always be suspected of when having a patient with Hypercalcemia as a dominant Clinical symptom. Hypercalcemia causes an increased gastric secretion, so in those patients the ulcer is present four times more frequently than in the general population [5].

Renal symptoms are polyuria and polydipsia. Diabetes hypercalcemicus must be investigated in any case of polyuria, especially when diabetes mellitus, diabetes insipidus, and tubulopathies have been excluded. Diuresis attributable to Hypercalcemia is accompanied by potassium loss; Hypercalcemia generally leads to hypokalemia. When Hypercalcemia reaches a critical level $(>4 \mathrm{mM})$, two organs are at risk for decompensation. Polyuria may develop into oliguria and finally anuria, especially in case of exsiccosis. Untreated Hypercalcemia renal insufficiency is lethal. The other organ at risk is the brain. Psychological disturbances may develop into somnolence and finally coma. For all patients with comas of questionable cause, a calcium-related coma must be excluded [6].

The following diagnostic program is recommended:

(1) Careful history and examination.

(2) X-rays of the head, thorax, vertebral column, pelvis, and long bones, to exclude osteolytic lesions attributable to pHPT, metastases, myeloma, or lung cancer.

(3) Ultrasound examination of the abdominal organs, to exclude hepatic, pancreatic, renal, or gynecologic tumors (occasionally, kidney stones or nephrocalcinosis indicates $\mathrm{pHPT}$ ).

(4) Laboratory studies such as phosphate, potassium, creatinine, urea, alkaline phosphates, sedimentation rate, and proteinuria measurements (Hypercalcemia is known) and blood smears. Fast PTH measurement is only occasionally available. If all findings are generally normal and no tumor is found, pHPT should be suspected. Ultrasound examination of the neck region is recommended; it reveals a hypoechogenic nodule, consistent with a parathyroid adenoma, in approximately two-thirds of cases. Experienced surgeons are content with this result, but inexperienced surgeons are not more satisfied after computed tomography and magnetic resonance imaging examinations, which cause further delay. During this diagnostic procedure, the patient should be receiving symptomatic treatment (see below). The combination of calcium lowering within 12 to $24 \mathrm{~h}$ and emergency diagnostic testing should permit surgical treatment of pHPT within $24 \mathrm{~h}$.

\section{Conclusions}

Hypercalcemic crisis is a life-threatening condition that is currently rather rare; however, it presents the risk that medical action may be too slow. Most cases of hypercalcemic crisis are attributable to decompensating pHPT. They require neck surgery performed by an experienced endocrine surgeon.

Within 12 to $24 \mathrm{~h}$, two problems must be solved. On one hand, a short diagnostic program should lead to the exclusion of neoplasias producing Hypercalcemia. On the other hand, those hours should also be used to lower serum calcium levels. One method is forced diuresis combined with the use of highly potent bisphosphonates; in cases of impaired renal function, calcium-free hemodialysis is the treatment of choice. Hypercalcemic crisis should be treated in a unit with appropriate expertise.

\section{References}

1. Fraser WD. (2009). Hyperparathyroidism. Lancet. 374(9684):145-158.

2. Wermers RA, Khosla S, Atkinson EJ, Hodgson SF, O'Fallon WM, Melton LJ 3rd. (1997). The rise and fall of primary hyperparathyroidism: a population-based study in Rochester, Minnesota, 1965-1992. Ann Intern Med. 126: 433-440.

3. Catherine Cormier, Jean-Claude Souberbielle, André Kahan. (2004). Primary hyperparathyroidism and osteoporosis in 2004. Joint Bone Spine. 71(3):183-189.

4. Allan E. Siperstein and Mira Milas. (2012). Comprehensive Parathyroidectomy for the treatment of PHP. Mastery of Surgery. 39: 486.

5. Cinamon U, Turcotte R. (2006). Primary hyperparathyroidism and malignancy: "Studies by nature". Bone. 39(2):420-423.

6. Dr. Reinhard Ziegler. (2001). Medizinische Universitätsklinik und Poliklinik, Bergheimer Strasse 58, D-69115 Heidelberg, Germany. Hypercalcemic Crisis. 